\title{
Pathological findings in kidneys of acromegalic patients
}

Sylvère Störmann, Christine Pichl, Matthias Pichler, Julia Mestek, Marc Albersmeyer, Anna Pallauf, Jochen Schopohl.

Medizinische Klinik und Poliklinik IV, Klinikum der Universität München, Ziemssenstr. 1, 80336 München (Munich, Germany)

\section{Introduction}

Acromegaly is an endocrinopathy that affects many different organ systems and leads to multiple comorbidities. Data on morphologic pathology of kidneys in acromegaly is scarce.

\section{Methods}

We investigated 36 acromegalic patients (20 male, 16 female, mean age $57.4 \pm 12.5$ years) presenting in our outpatient clinic. We evaluated each patient's kidneys by ultrasound, measuring organ dimensions and volume as well as noting any pathologic findings.

\section{Results}

With latest recommendations on criteria for cure acromegaly was considered biochemically controlled in 19 patients (53\%). 8 patients were partially controlled (IGF-I within 30\% of the upper limit of normal) and 9 patients biochemically active. Mean duration of disease was $16.6 \pm 10.4$ years.

Renomegaly was found in 3 patients $(8 \%)$. A total of 33 simple cysts were found in 14 patients $(39 \%) .3$ of those patients had simple cysts in both kidneys. 3 patients $(8 \%)$ presented with complex renal masses of which one turned out to be an early stage renal cell carcinoma ( $\mathrm{pT} 1 \mathrm{~N} 0 \mathrm{M} 0$ ). Microscopic nephrocalcinosis was detected in 6 patients $(17 \%)$, and kidney stones in two patients $(6 \%)$. Furthermore, we found bilateral obstructive uropathy and one duplex kidney in one patient each. Overall, 24 patients $(67 \%)$ presented with pathological or anomalous findings.

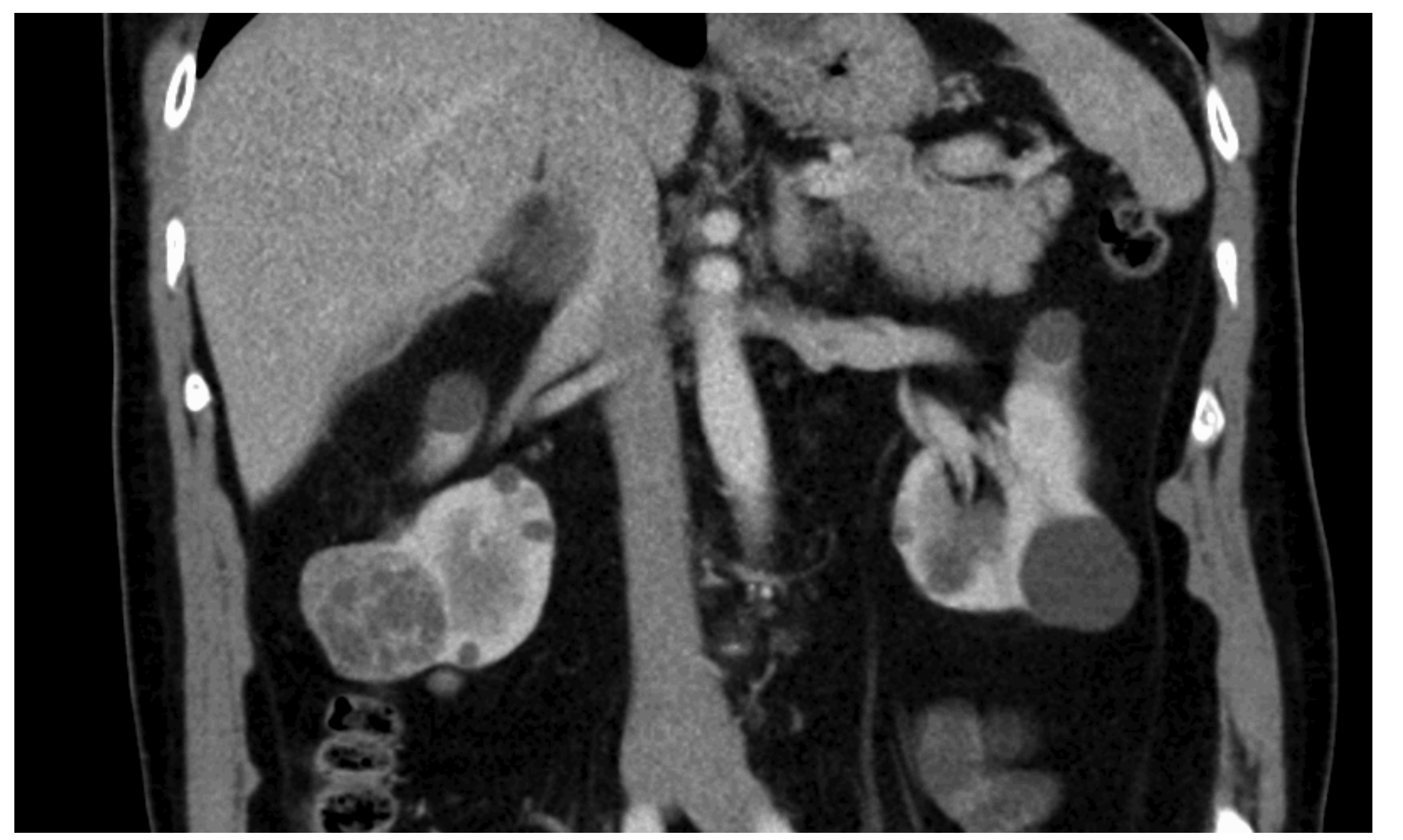

Fig. 1: The figure shows a CT scan (slice in coronal plane) of the abdomen of one of our patients. He presented with multiple simple as well as complex cysts. Under suspicion of a renal cell carcinoma surgery was performed. Histologic examination confirmed an RCC, classified as PT1N0M0.

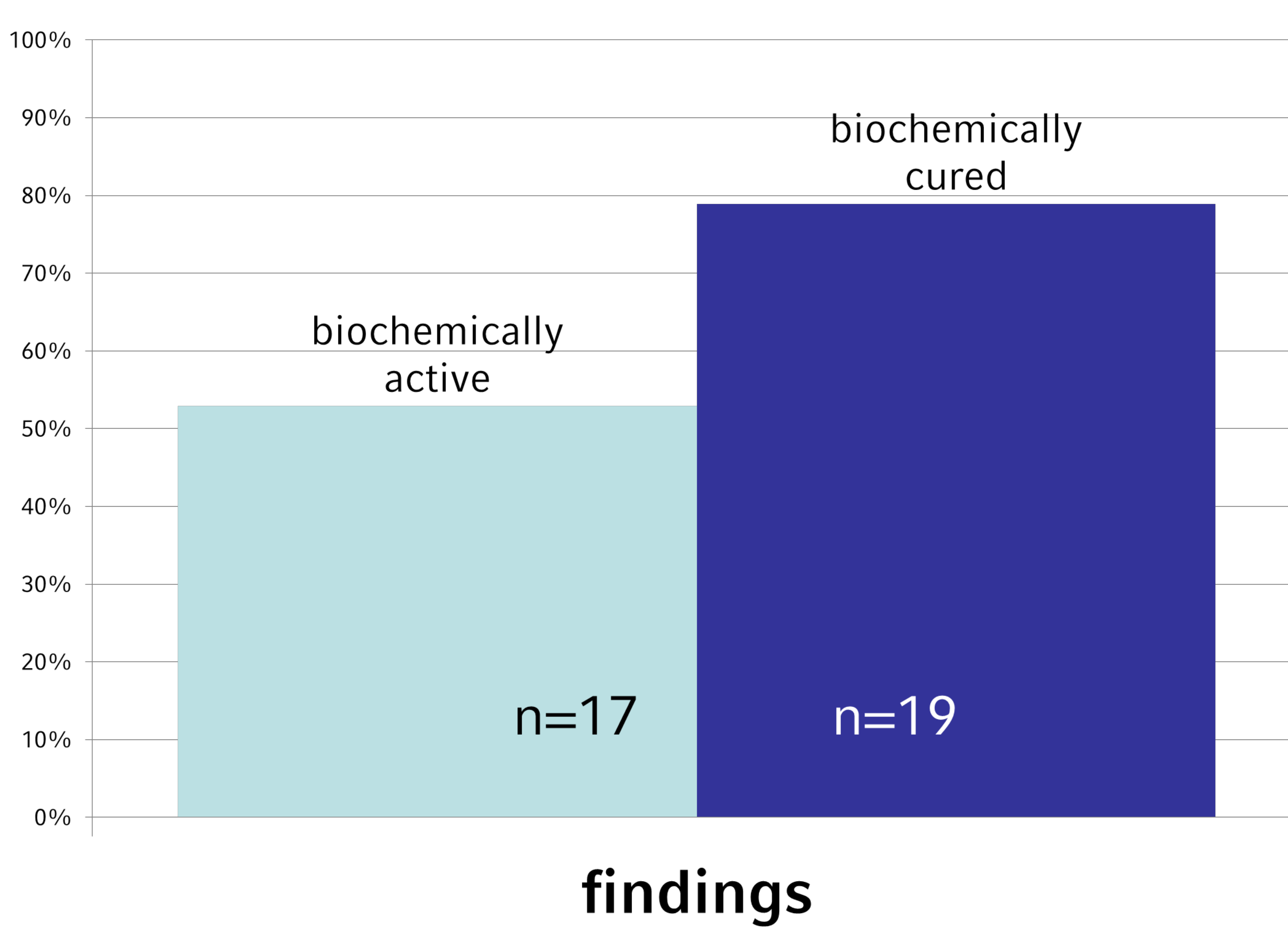

Fig. 2: Pathological and anomalous findings were discovered in a total of 24 patients. Patients considered [biochemically] cured had more findings than biochemically active patients.

\section{Conclusion}

We found a high prevalence of pathological ultrasonographic findings in a sample of 36 acromegalic patients. Biochemically cured patients did not yield less findings. Simple and complex renal cysts and nephrocalcinosis were more frequent than described in the literature for non-acromegalic patients. Further research is needed to better quantify our findings and to allow for sub-group analysis. 